\title{
Influencias sociales en un modelo de insatisfacción corporal, preocupación por el peso y malestar corporal en mujeres mexicanas
}

\author{
Karina Sugeyl Venegas-Ayala; Mónica Teresa González-Ramírez
}

\begin{abstract}
How to cite this article:
Venegas-Ayala, K. S., \& González-Ramírez, M. T. (2020). Social influences in a model of body dissatisfaction, weight worry and bodily discomfort in Mexican women. Acta Colombiana de Psicología, 23(1), 7-17. doi: http://www.doi. org/10.14718/ACP.2020.23.1.2
\end{abstract}

Recibido, octubre 16/2018; Concepto de evaluación, abril 9/2019; Aceptado, junio 17/2019

\author{
Karina Sugeyl Venegas-Ayala* \\ Universidad Autónoma de Nuevo León, México \\ ORCID: https://orcid.org/0000-0002-7766-2446 \\ Mónica Teresa González-Ramírez \\ Universidad Autónoma de Nuevo León, México \\ ORCID: https://orcid.org/0000-0002-9058-9626
}

\begin{abstract}
Resumen
El objetivo del presente estudio fue analizar el grado explicativo de las variables "influencia de la publicidad", "mensajes verbales", "modelos sociales" y "situaciones sociales" frente a la insatisfacción corporal, la preocupación por el peso y el malestar corporal en una muestra seleccionada por conveniencia de 206 mujeres mexicanas con promedio de edad de 22.12 años $(D T=4.21)$. Específicamente, se utilizó un modelamiento de ecuaciones estructurales para identificar los efectos directos e indirectos de las variables independientes sobre las dependientes en tres modelos hipotéticos propuestos, y como resultados se encontró que el conjunto de variables tuvo un valor explicativo de $79 \%$ para el modelo planteado para la insatisfacción corporal, con buenos indicadores de bondad de ajuste $\left(\chi^{2} / g l=.877 ; G F I=.982 ; C F I=.978 ; A G F I=.980\right.$; $S R M R=.068 ; R M S E A=.079)$; de $62 \%$ para el modelo de preocupación por el peso, con una adecuada bondad de ajuste $\left(\chi^{2} / g l=1.556 ; G F I=.981 ; C F I=.975 ; A G F I=.978 ; S R M R=.068 ; R M S E A=.080\right) ; \mathrm{y}$ de $72 \%$ para el modelo de malestar corporal, con buenos valores de bondad de ajuste $\left(\chi^{2} / g l=.173 ; G F I=.976 ; C F I=.969 ; A G F I=.972 ; S R M R=.062\right.$; $R M S E A=.072$ ). Se concluye que las influencias sociales tienen un impacto significativo en la imagen corporal y que los mensajes verbales presentan un mayor impacto en las variables estudiadas.
\end{abstract}

Palabras clave: imagen corporal, insatisfacción corporal, preocupación por el peso, malestar corporal, modelo explicativo.

\section{Social influences in a model of body dissatisfaction, weight worry and bodily discomfort in Mexican women}

Abstract

\begin{abstract}
The aim of this study was to analyze the explanatory level of the variables advertising influence, verbal messages, social models and social situations as regards body dissatisfaction, weight worry, and bodily discomfort. The study was conducted in a convenience sample of 206 Mexican women with an average age of 22.12 years $(S D=4.21)$. Structural equation modeling was used to analyze the direct and indirect effects of the independent variables on the dependent ones in three hypothetical models proposed. In the case of the model proposed for body dissatisfaction, it was found that the set of variables had $79 \%$ of variance explained and showed adequate goodness-of-fit indices $\left(\chi^{2} / g l=.877 ; G F I=.982 ; C F I=.978\right.$; $A G F I=.980 ; S R M R=.068 ; R M S E A=.079)$. The model for weight worry had $62 \%$ of variance explained and an acceptable goodness of fit $\left(\chi^{2} / g l=1.556 ; G F I=.981 ; C F I=.975 ; A G F I=.978 ; S R M R=.068 ; R M S E A=.080\right)$. Finally, the model for bodily discomfort had $72 \%$ of variance explained and showed adequate goodness of fit $\left(\chi^{2} / g l=.173 ; G F I=.976 ; C F I=\right.$ $.969 ; A G F I=.972 ; S R M R=.062 ; R M S E A=.072)$. It is concluded that social influences have a significant impact on body image. Verbal messages had the strongest impact on the variables studied.

Key words: Body image, body dissatisfaction, weight worry, bodily discomfort, explanatory model.
\end{abstract}

Carlos Canseco n. ${ }^{\circ}$ 110, Mitras Centro, Monterrey, México. Tel.: (+5281) 83294050. karina.venegasay@uanl.edu.mx 


\section{Introducción}

El concepto de "imagen corporal" ha cambiado constantemente a lo largo de la historia debido a que su descripción depende de factores relacionados con la época, la cultura, los procesos biológicos y la globalización (Gonçalves \& Bedin, 2016; Grogan, 2008). Por esta razón, en la actualidad, y como resultado de la evolución del contexto social, se ha planteado como necesario el tener un cuerpo sin defectos y extremadamente delgado, ya que esto equivaldría a tener un "cuerpo perfecto" (Amaya, Álvarez \& Mancilla, 2010).

Teniendo esto en cuenta, las mujeres que quieren alcanzar dicho ideal de belleza tienden a modifican sus esquemas cognitivos — reforzados por la concepción social—, y con el paso del tiempo estas ideas se adoptan y se vuelven conductas que ponen en riesgo su salud física (Jones \& Morgan, 2010; Neziroglu, Khemlani-Patel \& Veale, 2008; Ramírez et al., 2015; Rodríguez \& Cruz, 2008), aspecto que incrementa la probabilidad de padecer un Trastorno de Conducta Alimentaria (TCA) (Gerbasi et al., 2014; Jauregui \& Bolaños, 2011).

Debido a que las influencias sociales de la actualidad le otorgan una gran importancia a alcanzar un cuerpo socialmente aceptable, en distintos trabajos (Vázquez, Álvarez \& Mancilla, 2000; Vázquez et al., 2011) se ha encontrado que el no lograr dicho objetivo puede generar una preocupación por el peso; que si dicha preocupación es constante, esta puede ocasionar malestar corporal; que si dicho malestar no es resuelto a tiempo, es probable que propicie la insatisfacción corporal; y que tal insatisfacción pude provocar baja autoestima y baja percepción de apoyo social en las mujeres.

Adicional a esto, la retroalimentación hecha socialmente crea una percepción unilateral, ya que en esta se plantea que una mujer con buena imagen corporal es percibida como una persona agradable, exitosa, guapa y segura. Por esta razón, tener el "cuerpo ideal" toma una gran importancia en la cultura actual, y esto motiva a que las mujeres terminen decidiendo realizar largas jornadas de ejercicio, que asuman dietas extremas y que tomen laxantes - entre otras conductas nocivas - a pesar de saber las implicaciones negativas de dichas prácticas en su salud en general (Herraiz-Serrano et al., 2015; Trejo-Ortíz, Mollinedo, Araujo, Valdez-Esparza \& Sánchez, 2016).

Ahora bien, la relación entre la imagen corporal y las influencias sociales aparece cada vez que la apariencia física evoluciona, y este cambio suele deberse a diversos factores, como lo son los medios de comunicación, los medios sociales, los mensajes verbales y las relaciones sociales, entre otros (Cash, 1990). Sin embargo, aún no se han determinado las variables que tienen mayor impacto, ni cómo es que se correlacionan entre sí y tampoco la forma en que influyen sobre la imagen corporal (Balantekin, Birch \& Savage, 2018).

Adicional a lo anterior, se ha encontrado que la influencia social tiene mayor relevancia en entornos económicamente desarrollados, ya que en estos los medios de comunicación masivos y el contexto social juegan un papel importante en la divulgación de cuerpos idealizados y extremadamente delgados (Bearman, Presnell \& Martínez, 2006; Ferguson, Muñoz, Garza \& Galindo, 2014; Frederick, Forbes, Grigorian \& Jarcho 2007; Moreno-Murcia, Marcos-Pardo \& Huéscar, 2016; Vaquero-Cristóbal, Alacid, Muyor \& López-Miñarro, 2013). Asimismo, es de esperarse que en estos contextos el modelamiento de conducta y los mensajes verbales de iguales o familiares influyan en el desarrollo del malestar corporal (Balantekin et al., 2018).

Asimismo, se ha encontrado que la opinión del contexto social inmediato refuerza las conductas creadas para alcanzar el cuerpo deseado, a la vez que alienta a la comparación entre pares, lo que lleva a que las mujeres desarrollen un sentido de logro cuando su cuerpo es el más elogiado por sus amigos y familiares (Cruwys, Leverington \& Sheldon, 2016; Francisco, Narciso \& Alarcão, 2013; Gerbasi et al., 2014; Saffon \& Saldarriaga, 2014; Wasylkiw \& Williamson, 2013); sin embargo, en este aspecto es importante tener en cuenta que el reconocimiento social por tener un cuerpo perfecto es más valioso cuando la comparación es con un modelo real y no con uno idealizado - como los que presentan en los medios de comunicación-.

De igual forma, la publicidad también cumple un papel importante en la influencia social, pues en ella se muestran cuerpos cada vez más inalcanzables, lo que puede influir determinantemente en la insatisfacción de la imagen corporal $\mathrm{y}$ en el desarrollo o fomento de ideas y comportamientos que desencadenen síntomas indeseables, o, incluso, un diagnóstico de TCA (Losada, Leonardelli \& Magliola, 2015; Mas-Manchón et al., 2015).

Teniendo lo anterior en consideración, el estudio de la influencia de los pares, de la publicidad y de los medios de comunicación sobre la insatisfacción corporal se encuentra completamente justificado, y es en este sentido que en diversas investigaciones se ha encontrado una mayor influencia de los medios sociales y de los pares, aunque los resultados con respecto a la influencia de la publicidad no son concluyentes (Fardouly, Pinkus \& Vartanian, 2017; Ferguson et al., 2014; Rodgers, Paxton \& McLean, 2014). Asimismo, en otras investigaciones el enfoque ha sido explicar la forma en que la interacción de la insatisfacción corporal con los factores sociales provoca mala percepción 
corporal, autoestima contingente y altos índices de comparación (Bailey \& Ricciardelli, 2010; Ferguson, Winegard \& Winegard, 2011; Salazar-Mora, 2008).

Por otra parte, este problema de la insatisfacción corporal se está incrementando de tal manera que en países en vías de desarrollo, como en México, es cada vez más común encontrar que las adolescentes tienden a desarrollar preocupación excesiva por la imagen corporal, distintos rasgos que preceden la génesis de un TCA (Vaquero-Cristóbal et al., 2013), y que las mujeres están siendo afectadas por los estereotipos de la extrema delgadez, todo lo cual puede provocar una baja autoestima y un mayor deseo de ser delgadas (Botella, Ribas \& Ruiz, 2009; Vaquero-Cristóbal et al., 2013).

Finalmente, se puede concluir entre las variables más importantes para explicar esta problemática se pueden encontrar: (a) la insatisfacción corporal, que se presenta si se interioriza el cuerpo ideal y, al compararse con ese ideal, la persona concluye que su cuerpo discrepa con él (Acosta \& Gómez, 2003); (b) la preocupación por el peso, que se refiere al peso del individuo en relación con la ingesta y la preocupación desarrollada por los aspectos antiestéticos de la obesidad (Vázquez et al., 2011); (c) el malestar corporal, que aparece cuando se siente ansiedad al enfrentar situaciones que cuestionan la belleza del cuerpo (Vázquez et al., 2000); y (d) la influencia de las situaciones sociales, que refiere a la presión social subjetiva experimentada en situaciones alimentarias y a la aceptación social atribuida a la delgadez (Vázquez et al., 2000).

Teniendo todo lo anterior en cuenta, y sabiendo que cada vez son más las mujeres mexicanas que cambian los esquemas de pensamiento y desarrollan conductas para alcanzar una imagen corporal ideal, la forma en que se relaciona la imagen corporal con el contexto social es un aspecto que merece toda la atención en términos de investigación, y es por esto que en el presente estudio se planteó como objetivo emplear un método de ecuaciones estructurales que permitiera conocer a profundidad la relación entre dichas

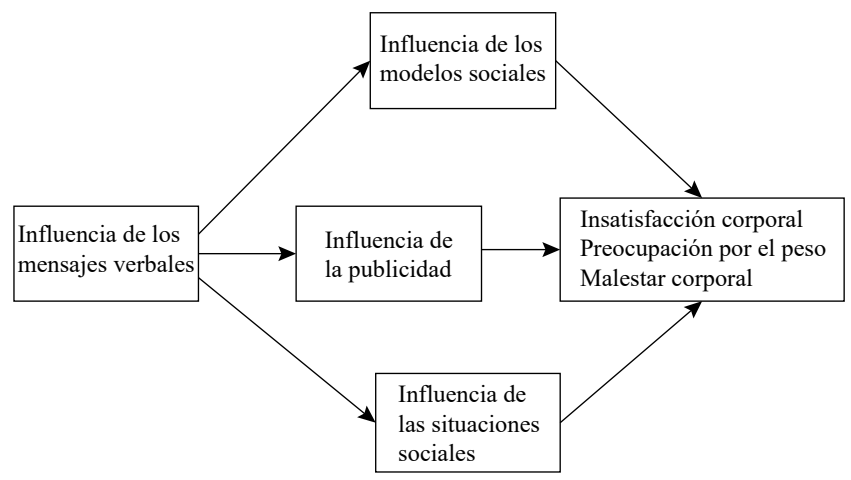

Figura 1. Modelos estructurales propuestos. variables. En la Figura 1 se pueden observar los modelos teóricos propuestos para la presente investigación.

\section{Método}

\section{Diseño}

La presente investigación tuvo un diseño no experimental, correlacional-explicativo y de corte transversal, en el que el método de obtención de información fue la aplicación de una encuesta de dos instrumentos de medición (Thompson, Diamond, McWilliam, Snyder \& Snyder, 2005).

\section{Participantes}

Se hizo uso un muestreo incidental con el que se obtuvo una muestra de 206 mujeres, con promedio de edad de 22.12 años $(D T=4.21)$, residentes de la ciudad de Monterrey, México. Del total de la muestra, 125 (60.7\%) reportaron ser estudiantes, 72 (35\%) dijeron ser empleadas, y nueve, (4.4\%) amas de casa. En cuanto a estado civil, 113 $(54.9 \%)$ reportaron estar solteras sin pareja, 69 (33.5\%) mencionaron ser solteras con pareja, 23 (11.2\%) dijeron estar casadas, y una $(0.5 \%)$ reportó ser divorciada. Como criterios de inclusión se planteó que las participantes fueran mujeres mexicanas y que tuvieran de 14 a 30 años de edad; y como criterios de exclusión se tuvo el no cumplir con el rango de edad o el ser de sexo masculino.

\section{Instrumentos}

Cuestionario de la Figura Corporal (BSQ; Cooper, Taylor, Cooper \& Fairbum, 1987). Se utilizó la versión en español de Raich et al. (1996), validada en Colombia por Castrillón, Luna, Avendaño y Pérez-Acosta (2007). Este cuestionario cuenta con 34 ítems -20 evalúan la insatisfacción corporal y 14 la preocupación por el peso-que se responden por medio de una escala tipo Likert de seis opciones ( $1=$ "nunca" a $6=$ "siempre"). En su versión original, este instrumento contó con una consistencia interna de $\alpha=.96$, mientras que en el presente estudio se obtuvo un $\alpha=.96$ y un $\omega=.95$.

Cuestionario de Influencias del Modelo Estético Corporal (CIMEC-26; Toro, Salamero \& Martínez, 1994). Se utilizó una versión abreviada de 26 ítems, de los cuales ocho evalúan el malestar corporal, ocho la influencia de la publicidad, tres la influencia de los mensajes verbales, cuatro la influencia de las situaciones sociales y tres la influencia de los modelos sociales. Todos los reactivos se responden por medio de 
una escala tipo Likert de tres opciones $(0=$ "no, nunca" a 2 = "sí, siempre"). La consistencia interna en la versión original de este instrumento fue de $\alpha=.93$ en pacientes con anorexia y de $\alpha=.91$ en población con ausencia de TCA. En el presente estudio se obtuvo una consistencia interna de $\alpha=.93$ y un $\omega=.91$.

\section{Procedimiento}

Se realizó una convocatoria abierta por medio de redes sociales para participar de manera voluntaria en el estudio. Después de esta convocatoria, se realizó un contacto presencial con las participantes, se obtuvo el correspondiente consentimiento informado, y posteriormente, desde un equipo de cómputo proporcionado por el equipo de investigación, se les indicó que abrieran un enlace en internet que les daba acceso a los instrumentos. En el caso de las participantes menores de edad (29 en total), se solicitó el consentimiento informado del padre o tutor al momento de la aplicación de los instrumentos.

A todas las participantes se les garantizó absoluta confidencialidad y que los resultados obtenidos solo serían utilizados con fines académicos. Además, se dio la opción de atención psicológica en caso de que alguna participante así lo solicitara. Cabe mencionar que no se dio ninguna remuneración monetaria o material a cambio de la participación en la presente investigación, y que esta investigación fue aprobada en sus aspectos técnicos y éticos por el Comité Doctoral de la Facultad de Psicología de la Universidad Autónoma de Nuevo León.

\section{Análisis de datos}

Se utilizó el IBM SPSS 24 para los análisis descriptivos, correlaciones ( $r$ de Pearson) y fiabilidad por alfa de Cronbach $(\alpha)$, y el AMOS 24 para calcular el coeficiente de normalidad multivariada (Mardia) y ejecutar el análisis de modelamiento de ecuaciones estructurales.

Específicamente, para establecer el valor de fiabilidad omega $(\omega)$ se utilizó la expresión matemática encontrada en McDonald (1999) — no se tuvieron casos perdidos en la muestra- En cada una de las pruebas se calcularon las puntuaciones típicas para determinar, mediante el puntaje $Z$, la existencia de casos atípicos que tuvieran un valor de \pm 3 . Del mismo modo, se realizó el procedimiento de distancia Mahalanobis $\left(D^{2}\right)$ para la búsqueda de casos atípicos multivariados que superaran el límite de significación $(p<.001)$ (Tabachnick \& Fidell, 2013). Tras revisar ambos estadísticos, se estableció la inexistencia de outliers en la muestra. También, se determinó el coeficiente de Mardia para confirmar la distribución normal multivariada, el cual resultó adecuado $(<70)$ en cada modelo $\left(\mathrm{M}_{1=} 45.70\right.$,
$\mathrm{M}_{2=}$ 22.17, $\mathrm{M}_{3=}$ 19.33; Rodríguez \& Ruiz, 2008). Por otra parte, para confirmar los modelos teóricos propuestos, se utilizó la técnica de modelamiento de ecuaciones estructurales - los análisis estadísticos se efectuaron con el criterio de máxima verosimilitud y no se eliminó ningún ítem de las escalas utilizadas en la investigación-.

Finalmente, para probar la bondad de ajuste de cada modelo se consideraron siete índices: la razón entre la chi-cuadrara y los grados de libertad $\left(\chi^{2} / g l\right)$, el índice de bondad de ajuste (GFI), el índice ajustado de bondad de ajuste $(A G F I)$, el índice de ajuste comparativo $(C F I)$, el error cuadrático medio de aproximación ( $R M S E A)$, la raíz normalizada cuadrada media residual $(S R M R)$ y la razón de parsimonia $(P R)$; y para dichos coeficientes se esperaron valores de $\chi^{2} / g l \leq 2, G F I$ y $C F I \geq .95, A G F I \geq .90, R M S E A$ y $S R M R \leq .08$ y $P R>.75$, todos indicadores de un buen ajuste a los datos (Byrne, 2016).

Los modelos propuestos en la Figura 1 tienen como constante las mismas cuatro variables independientes -influencia de la publicidad, influencia de los mensajes verbales, influencia de los modelos sociales e influencia de las situaciones sociales - y el único cambio de un modelo a otro con respecto al esquema teórico fue la variable dependiente, es decir, la insatisfacción corporal, la preocupación por el peso y el malestar corporal. El estudio consistió únicamente en medidas de autorreporte y no se obtuvieron datos antropométricos como peso o altura.

\section{Resultados}

En el presente apartado se muestran las correlaciones, las medidas de tendencia central y los valores de fiabilidad de los instrumentos utilizados, seguidos por la bondad de ajuste global de los modelos propuestos y, por último, los efectos directos e indirectos y los valores de la varianza explicada.

\section{Estadísticos descriptivos, correlaciones y fiabilidad}

Antes de realizar el modelamiento de ecuaciones estructurales se llevó a cabo un análisis de correlación de Pearson entre el total de variables estudiadas, se estimaron las medias $(M)$ y desviaciones estándar o típicas $(D T)$ de las mismas, y se reportaron los valores de fiabilidad por cada subescala (véase Tabla 1).

Es importante señalar que todas las variables correlacionaron positivamente y de manera significativa a un nivel de $p<.01$; que las correlaciones más altas fueron entre la insatisfacción corporal y la preocupación por el peso $(r=.871, p<.01)$, entre la insatisfacción corporal y el malestar corporal $(r=.848, p<.01)$, y entre la 
Tabla 1.

Correlaciones, medias, desviaciones típicas, $\alpha$ y $\omega$ de las variables investigadas

\begin{tabular}{|c|c|c|c|c|c|c|c|}
\hline Variable & V1 & $\mathrm{V} 2$ & $\mathrm{~V} 3$ & $\mathrm{~V} 4$ & V5 & V6 & V7 \\
\hline V1 & 1 & $.871^{*}$ & $.848^{*}$ & $.728 *$ & $.360^{*}$ & $.485^{*}$ & $.632 *$ \\
\hline $\mathrm{V} 2$ & - & 1 & $.826^{*}$ & $.685^{*}$ & $.441 *$ & $.513 *$ & $.480^{*}$ \\
\hline V3 & - & - & 1 & $.712 *$ & $.414^{*}$ & $.522 *$ & $.569 *$ \\
\hline V4 & - & - & - & 1 & $.566^{*}$ & $.448 *$ & $.475^{*}$ \\
\hline V5 & - & - & - & - & 1 & $.392 *$ & $.320 *$ \\
\hline V6 & - & - & - & - & - & 1 & $.483^{*}$ \\
\hline V7 & - & - & - & - & - & - & 1 \\
\hline$M$ & 42.46 & 42.03 & 6.25 & 2.87 & 1.66 & 3.15 & 1.64 \\
\hline$D T$ & 17.093 & 15.226 & 4.253 & 3.606 & 1.621 & 1.724 & 1.464 \\
\hline$\alpha$ & .940 & .760 & .856 & .908 & .768 & .549 & .637 \\
\hline$\Omega$ & .930 & .740 & .861 & .911 & .757 & .529 & .668 \\
\hline
\end{tabular}

Nota. ${ }^{*} p<.01 . \mathrm{V} 1$ = insatisfacción corporal, V2 = preocupación por el peso, V3 = malestar corporal, V4 = influencia de la publicidad, V5 = influencia de los mensajes verbales, V6 = influencia de los modelos sociales, V7 = influencia de las situaciones sociales, $M=$ media, $D T=$ desviación típica, $\alpha=$ alfa de Cronbach, $\Omega=$ omega de McDonald.

preocupación por el peso y el malestar corporal $(r=.826$, $p<.01)$; y que la correlación más baja fue la influencia de los mensajes verbales y la influencia de las situaciones sociales $(r=.320, p<.01)$.

Adicional a esto, las correlaciones entre las variables dependientes fueron altas $(r \geq .80)$, lo que significa que existe multicolinealidad y que, por tanto, podría haber una afectación directa en los indicadores de bondad de ajuste y grado explicativo de los modelos propuestos (Kline, 2015). Teniendo esto en cuenta, la alta relación estadística entre los constructos insatisfacción corporal, preocupación por el peso y malestar corporal debe ser diferenciada de manera teórica, pues al no hacerlo se obtendrían errores de categorización y de inconsistencia estadística que podrían provocar una mala interpretación de los resultados (Mancilla, Vázquez, Mancilla, Amaya \& Álvarez, 2012). Debido a lo anterior, se justifica el uso de modelos separados para cada una de las variables dependientes para, con ello, poder conocer la influencia que tienen sobre ellas la publicidad, los mensajes verbales, los modelos y las situaciones sociales.

\section{Bondad de ajuste de los modelos propuestos}

Los parámetros resultantes indican que los tres modelos propuestos cuentan con buena bondad de ajuste en los siete indicadores reportados (véase Tabla 2), y es importante mencionar que no se correlacionó ningún error de covarianza ni se eliminó algún ítem para mejorar los modelos.

Con respecto a los resultados, cabe destacar que el valor de $\chi^{2} / g l$ para los modelos $\mathrm{M}_{1}$ y $\mathrm{M}_{3}$ fue menor a uno, lo que podría ser un indicador de sobreestimación (Escobedo, Hernández, Estebané \& Martínez, 2016). Sin embargo, esta hipótesis fue rechazada, puesto que los tres modelos estudiados obtuvieron una $P R$ mayor a .75 (Byrne, 2016;
James, Mulaik \& Brett, 1982; Mulaik et al., 1989). Debido a que la aceptación de cualquier modelo es dada por múltiples indicadores (Byrne, 2016), es posible afirmar que los modelos propuestos se ajustan de manera adecuada a los datos empíricos.

Efectos directos e indirectos entre las variables de estudio

En las figuras 2, 3 y 4 se muestran los efectos directos, $\mathrm{y}$ en la Tabla 3 se pueden encontrar los efectos indirectos de los tres modelos propuestos.

Modelo para la insatisfacción corporal $\left(M_{1}\right)$. En el caso del modelo para la insatisfacción corporal (véase Figura 2), los parámetros propuestos obtuvieron en su mayoría un nivel de significación de $p<.01$, y como resultados se obtuvieron efectos directos de la influencia de la publicidad $(\beta=.39, p<.01) \mathrm{y}$ de las situaciones sociales $(\beta=.53$, $p<.01)$ en la insatisfacción corporal —el coeficiente de regresión para la influencia de los modelos sociales no fue significativo $(\beta=.11, p>.05)$-, así como efectos directos de la influencia de los mensajes verbales en la influencia de la publicidad $(\beta=.62, p<.01)$ y de la influencia de los mensajes verbales en las situaciones sociales $(\beta=.60$, $p<.01) \mathrm{y}$ en los modelos sociales $(\beta=.66, p<.01)$.

Por otra parte, la influencia de los mensajes verbales tuvo un efecto indirecto sobre la insatisfacción corporal, mediado por la influencia de la publicidad $(\beta=.31, p<.01)$; si se agrega la ruta de la influencia de las situaciones sociales, se obtiene un doble efecto mediador de los mensajes verbales sobre la insatisfacción corporal $(\beta=.64, p<.01)$; y al utilizar la ruta de la influencia de los modelos sociales se obtiene un efecto no significativo sobre la insatisfacción corporal $(\beta=.12, p>.05)$. Por su parte, la influencia de la 
Tabla 2.

Índices de bondad de ajuste para los modelos propuestos

\begin{tabular}{cccccccc}
\hline Modelo & $\chi^{2} / g l$ & GFI & CFI & AGFI & SRMR & RMSEA & PR \\
\hline $\mathrm{M}_{1}$ & .877 & .982 & .978 & .980 & .068 & .079 & .937 \\
$\mathrm{M}_{2}$ & 1.556 & .981 & .975 & .978 & .068 & .080 & .923 \\
$\mathrm{M}_{3}$ & .173 & .976 & .969 & .972 & .062 & .072 & .902 \\
\hline
\end{tabular}

Nota $. \mathrm{M}_{1}=$ modelo para la insatisfacción corporal, $\mathrm{M}_{2}=$ modelo para la preocupación por el peso, $\mathrm{M}_{3}=$ modelo para el malestar corporal. Valores que indican un buen ajuste a los datos: $\chi^{2} / g l \leq 3, G F I$ y $C F I \geq .95, A G F I \geq .90, R M S E A$ y $S R M R \leq .08, P R>.75$.

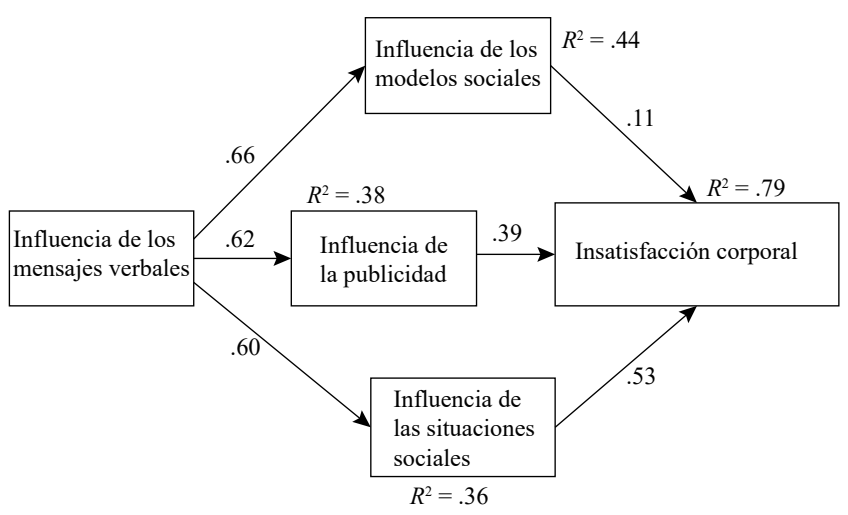

Figura 2. Modelo para la insatisfacción corporal $\left(\mathrm{M}_{1}\right)$.

publicidad tuvo un efecto indirecto sobre la insatisfacción corporal, mediado por la influencia de las situaciones sociales $(\beta=.39, p<.01)$; pero en el caso de la influencia de los modelos sociales el efecto no fue significativo $(\beta=.077$, $p>.05)$. Otros efectos indirectos encontrados fueron la influencia de los mensajes verbales en la influencia de las situaciones sociales $(\beta=.29, p<.01)$ y en la influencia de los modelos sociales $(\beta=.36, p<.01)$, utilizando como variable mediadora la influencia de la publicidad.

Por último, se encontró que la influencia de los mensajes verbales explicó el $38 \%$ de la varianza de la influencia de la publicidad, mientras que la influencia de los mensajes verbales y la influencia de la publicidad explicaron el $44 \%$ de la influencia de los modelos sociales y el $36 \%$ de la influencia de las situaciones sociales. En suma, la influencia de los mensajes verbales en mediación con la influencia de la publicidad $(\beta=.31, p<.01)$ y los mensajes verbales en mediación con la influencia de la publicidad y la influencia de las situaciones sociales $(\beta=.64, p<.01)$ explicaron el $79 \%$ de la varianza de la insatisfacción corporal.

Modelo para la preocupación por el peso $\left(M_{2}\right)$. Con respecto al modelo para la preocupación por el peso (véase Figura 3), las rutas propuestas obtuvieron en su mayoría un nivel de significación de $p<.01$, con excepción del par "situaciones sociales $\rightarrow$ preocupación por el peso", además de las vías "publicidad $\rightarrow$ modelos sociales $\rightarrow$ preocupación por el peso" y "mensajes verbales $\rightarrow$ publicidad $\rightarrow$ situaciones sociales $\rightarrow$ preocupación por el peso", que fueron significativas a nivel, $p<.05$.

Por otra parte, se encontraron efectos directos de la influencia de la publicidad $(\beta=.42, p<.01)$, las situaciones sociales $(\beta=.21, p<.05)$ y los modelos sociales $(\beta=.29$, $p<.01)$ en la preocupación por el peso. Otros efectos directos en este modelo fueron la influencia de los mensajes verbales en la influencia de la publicidad $(\beta=.63, p<.01)$, además de la influencia de los mensajes verbales en las situaciones sociales $(\beta=.60, p<.01) \mathrm{y}$ en los modelos sociales $(\beta=.66, p<.01)$. Estos últimos efectos presentaron poca o nula variabilidad respecto al modelo anterior $\left(\mathrm{M}_{1}\right)$.

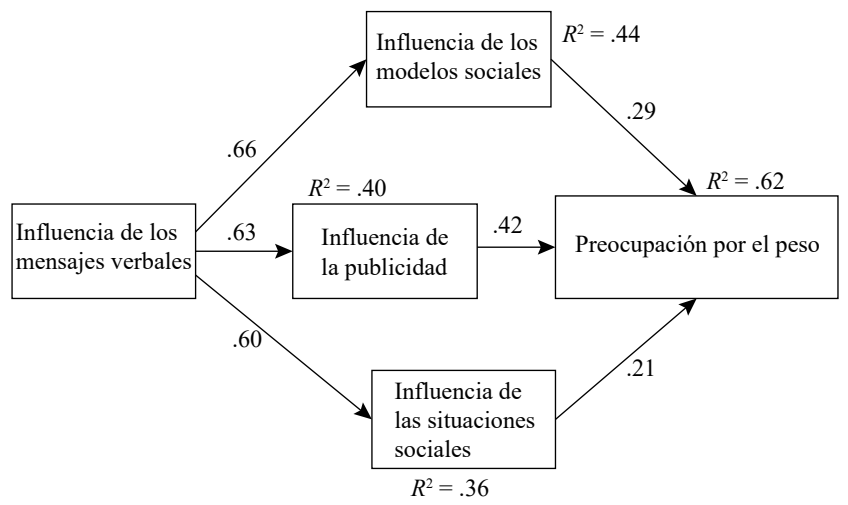

Figura 3. Modelo para la preocupación por el peso $\left(\mathrm{M}_{2}\right)$.

En lo que refiere a los efectos indirectos, la influencia de los mensajes verbales tuvo un efecto indirecto sobre la preocupación por el peso, mediado por la influencia de la publicidad ( $\beta=.52, p<.01)$; pero, si se agrega la ruta de la influencia de las situaciones sociales, se obtiene un doble efecto mediador de los mensajes verbales sobre la preocupación por el peso $(\beta=.37, p<.05)$; y al utilizar la ruta de la influencia de los modelos sociales también se obtiene un doble efecto mediador sobre preocupación por el peso $(\beta=.46, p<.01)$. Por su parte, la influencia de la publicidad tuvo un efecto indirecto sobre la preocupación por el peso, mediado por la influencia de las situaciones sociales $(\beta=.39, p<.01)$ y a su vez por la influencia de los modelos sociales $(\beta=.31, p<.05)$. Otros efectos indirectos encontrados fueron la influencia de los mensajes 
verbales en la influencia de las situaciones sociales $(\beta=.37$, $p<.01)$ y en la influencia de los modelos sociales $(\beta=.38$, $p<.01$ ), utilizando como variable mediadora la influencia de la publicidad.

Finalmente, se encontró que la influencia de los mensajes verbales explicó el $40 \%$ de la varianza de la influencia de la publicidad; que la influencia de los mensajes verbales explicó el $44 \%$ de la variable influencia de los modelos sociales y el $36 \%$ de la influencia de las situaciones sociales; $\mathrm{y}$, en conjunto, que la influencia de los mensajes verbales en mediación con la influencia de la publicidad $(\beta=.52$, $p<.01)$, los mensajes verbales en mediación con la influencia de la publicidad y la influencia de las situaciones sociales $(\beta=.37, p<.01)$ y los mensajes verbales en mediación con la influencia de la publicidad y la influencia de los modelos sociales $(\beta=.37, p<.01)$ explicaron el $62 \%$ de la varianza de la preocupación por el peso.

Modelo para el malestar corporal $\left(M_{3}\right)$. Con respecto al modelo para el malestar corporal (véase Figura 3), las rutas propuestas indicaron en su mayoría un nivel de significación de $p<.01$, con excepción del par "modelos sociales $\rightarrow$ malestar corporal", que tuvo un $p<.05$. En cuanto a los efectos directos, se encontró que la influencia de la publicidad $(\beta=.38, p<.01)$, las situaciones sociales $(\beta=.36, p<.01)$ y los modelos sociales $(\beta=.26, p<.05)$ tienen un efecto significativo en el malestar corporal; y que la influencia de los mensajes verbales tuvo un efecto directo en la influencia de la publicidad $(\beta=.63, p<.01)$, en las situaciones sociales $(\beta=.60, p<.01) \mathrm{y}$ en los modelos sociales $(\beta=.66, p<.01)$. Estos últimos valores presentaron poca o ninguna variabilidad respecto a los modelos anteriores $\left(\mathrm{M}_{1}\right.$ y $\left.\mathrm{M}_{2}\right)$.

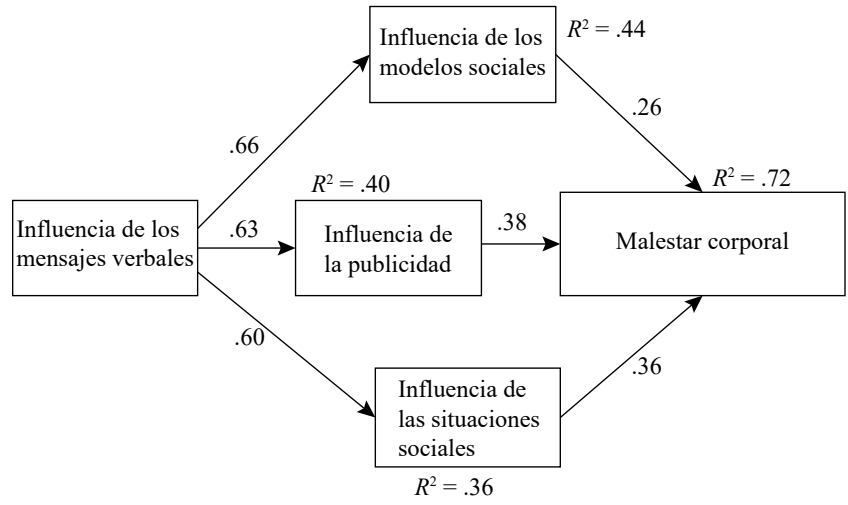

Figura 4. Modelo para el malestar corporal $\left(\mathrm{M}_{3}\right)$.

En lo que refiere a los efectos indirectos, la influencia de los mensajes verbales tuvo un efecto indirecto sobre el malestar corporal, mediado por la influencia de la publicidad $(\beta=.26, p<.01)$; pero, si se agrega la ruta de la influencia de las situaciones sociales, se obtiene un doble efecto mediador de los mensajes verbales sobre el malestar corporal $(\beta=.39, p<.01)$; y al utilizar la ruta de la influencia de los modelos sociales no se obtiene un efecto significativo sobre el malestar corporal $(\beta=.23, p>.05)$. Por su parte, la influencia de la publicidad tuvo un efecto indirecto sobre el malestar corporal, mediado por la influencia de las situaciones sociales $(\beta=.20, p<.01)$, pero no significativo mediante la influencia de los modelos sociales $(\beta=.16$, $p>.05)$. Otros efectos indirectos encontrados fueron la influencia de los mensajes verbales en la influencia de las situaciones sociales $(\beta=.29, p<.01)$ y en la influencia de los modelos sociales $(\beta=.38, p<.01)$, utilizando como variable mediadora la influencia de la publicidad.

Finalmente, se encontró que, al igual que en el modelo $\mathrm{M}_{2}$, la influencia de los mensajes verbales explicó el $40 \%$ de la varianza de la influencia de la publicidad, el $44 \%$ de la influencia de los modelos sociales y el $36 \%$ de la influencia de las situaciones sociales. En conjunto, la influencia de los mensajes verbales en mediación con la influencia de la publicidad $(\beta=.26, p<.01)$ y los mensajes verbales en mediación con la influencia de la publicidad y la influencia de las situaciones sociales $(\beta=.39, p<.01)$ explicaron el $72 \%$ de la varianza del malestar corporal.

\section{Discusión}

En general, cada uno de los modelos propuestos son evidencia de que las variables socioculturales influyen de manera significativa en la creación de una imagen corporal negativa; $y$, en particular, al evaluar el efecto de la influencia de los mensajes verbales sobre la influencia de la publicidad, los parámetros resultaron significativos en cada uno de los tres modelos propuestos. De este resultado en específico se infiere que para las mujeres es importante la retroalimentación de su contexto social, ya que de este depende la importancia y el enfoque que se le otorgue a los mensajes recibidos por los diferentes medios de comunicación (Fardouly et al., 2017; Ferguson et al., 2014; Rodgers et al., 2014).

Adicional a esto, en los modelos de insatisfacción corporal, malestar corporal y preocupación por el peso se encontró una influencia significativa de la publicidad, principalmente por su efecto directo con los modelos sociales, las situaciones sociales y los mensajes verbales, lo que coincide con hallazgos previos en donde se describe la influencia directa de los medios de comunicación y la publicidad en la imagen corporal de las mujeres (Fardouly et al., 2017; Ferguson et al., 2014; Rodgers et al., 2014). 
Tabla 3.

Efectos estandarizados indirectos entre las variables de estudio

\begin{tabular}{|c|c|}
\hline$M_{1}$ Insatisfacción corporal & Betas Estandarizadas $(\beta)$ \\
\hline Mensajes verbales $\rightarrow$ Publicidad $\rightarrow$ Insatisfacción corporal & $.31^{* * *}$ \\
\hline Mensajes verbales $\rightarrow$ Publicidad $\rightarrow$ Situaciones sociales & $.29^{* * *}$ \\
\hline Mensajes verbales $\rightarrow$ Publicidad $\rightarrow$ Modelos sociales & $.36^{* * *}$ \\
\hline Publicidad $\rightarrow$ Situaciones sociales $\rightarrow$ Insatisfacción corporal & $.34^{* * *}$ \\
\hline Publicidad $\rightarrow$ Modelos sociales $\rightarrow$ Insatisfacción corporal & $.077^{*}$ \\
\hline Mensajes verbales $\rightarrow$ Publicidad $\rightarrow$ Situaciones sociales $\rightarrow$ Insatisfacción corporal & $.64^{* * *}$ \\
\hline Mensajes verbales $\rightarrow$ Publicidad $\rightarrow$ Modelos sociales $\rightarrow$ Insatisfacción corporal & $.12^{*}$ \\
\hline \multicolumn{2}{|c|}{$R^{2}($ Situaciones sociales $)=.36 ; R^{2}($ Publicidad $)=.38 ; R^{2}($ Modelos sociales $)=.44 ; R^{2}($ Insatisfacción corporal $)=.79$} \\
\hline \multicolumn{2}{|c|}{$M_{2}$. Preocupación por el peso } \\
\hline Mensajes verbales $\rightarrow$ Publicidad $\rightarrow$ Preocupación por el peso & $.52^{* * *}$ \\
\hline Mensajes verbales $\rightarrow$ Publicidad $\rightarrow$ Situaciones sociales & $.37^{* * *}$ \\
\hline Mensajes verbales $\rightarrow$ Publicidad $\rightarrow$ Modelos sociales & $.38^{* * *}$ \\
\hline Publicidad $\rightarrow$ Situaciones sociales $\rightarrow$ Preocupación por el peso & $.21^{* * *}$ \\
\hline Publicidad $\rightarrow$ Modelos sociales $\rightarrow$ Preocupación por el peso & $.31^{* *}$ \\
\hline Mensajes verbales $\rightarrow$ Publicidad $\rightarrow$ Situaciones sociales $\rightarrow$ Preocupación por el peso & $.37^{* *}$ \\
\hline Mensajes verbales $\rightarrow$ Publicidad $\rightarrow$ Modelos sociales $\rightarrow$ Preocupación por el peso & $.46^{* * *}$ \\
\hline \multicolumn{2}{|c|}{$R^{2}($ Situaciones sociales $)=.36 ; R^{2}($ Publicidad $)=.40 ; R^{2}($ Modelos sociales $)=.44 ; R^{2}($ Preocupación por el peso $)=.62$} \\
\hline \multicolumn{2}{|c|}{$M_{3}$. Malestar corporal } \\
\hline Mensajes verbales $\rightarrow$ Publicidad $\rightarrow$ Malestar corporal & $.26^{* * *}$ \\
\hline Mensajes verbales $\rightarrow$ Publicidad $\rightarrow$ Situaciones sociales & $.29^{* * *}$ \\
\hline Mensajes verbales $\rightarrow$ Publicidad $\rightarrow$ Modelos sociales & $.38^{* * *}$ \\
\hline Publicidad $\rightarrow$ Situaciones sociales $\rightarrow$ Malestar corporal & $.20^{* * *}$ \\
\hline Publicidad $\rightarrow$ Modelos sociales $\rightarrow$ Malestar corporal & $.16^{*}$ \\
\hline Mensajes verbales $\rightarrow$ Publicidad $\rightarrow$ Situaciones sociales $\rightarrow$ Malestar corporal & $.39^{* * *}$ \\
\hline Mensajes verbales $\rightarrow$ Publicidad $\rightarrow$ Modelos sociales $\rightarrow$ Malestar corporal & $.23^{*}$ \\
\hline$R^{2}($ Situaciones sociales $)=.36 ; R^{2}($ Publicidad $)=.40 ; R^{2}($ Modelos sociales $)=.44$ & star corporal) $=.72$ \\
\hline
\end{tabular}

Nota. ${ }^{*} p>.05 ;{ }^{* *} p<.05 ;{ }^{* * *} \mathrm{p}<.01$.

Por otra parte, las situaciones sociales mostraron estar directamente relacionadas con la insatisfacción corporal, la preocupación por el peso y el malestar corporal, resultado que apoya la teoría, ya que se indica que en contextos en donde se persigue un canon de belleza poco alcanzable para las mujeres, este influye claramente en la mala percepción de su imagen corporal — sea esta patológica o normativa(Ferguson et al., 2014).

Específicamente, la variable modelos sociales solo mostró relación significativa con la preocupación por el peso, lo cual respalda la afirmación de que las mujeres solamente se fijan en modelos estéticos publicitarios cuando se trata de comparaciones con el peso, pero a la hora de realizar una comparación corporal general prefieren hacerlo con sus pares y no con modelos que consideran irreales (Brudzynski \& Ebben, 2010).

También, teniendo en cuenta que para los tres modelos se estudiaron los efectos considerando variables mediadoras — esto con la finalidad de saber cómo interactúan entre ellas con respecto a la formación de la imagen corporallos resultados obtenidos apoyan la propuesta teórica.

Así, en el primer modelo, que refiere a la insatisfacción corporal, se obtuvo una relación significativa entre los mensajes verbales, la publicidad, los modelos sociales y la insatisfacción corporal, lo cual muestra que los mensajes que se dan dentro del contexto social - por parte de padres, pares, pareja, compañeros, etc.- influyen en el tipo de publicidad que se elige ver, y esto a su vez influye en que se reproduzcan situaciones sociales que favorecen la insatisfacción corporal (Fardouly et al., 2017).

En el segundo modelo, que considera la preocupación por el peso, se encontró una relación entre los mensajes verbales, la publicidad, las situaciones sociales y la preocupación por el peso, lo cual evidencia que la interacción de los comentarios con respecto al peso, los comerciales que promueven la reducción de peso y las situaciones que promueven la figura delgada tendrán como resultado la preocupación por el peso (Caccavale, Farhat \& Iannotti, 
2012). Esta variable también mostró estar relacionada con los mensajes verbales, la publicidad y los modelos sociales, lo cual es congruente con investigaciones previas (Bailey \& Ricciardelli, 2010; Fardouly et al., 2017; Ferguson et al., 2011; Ferguson et al., 2014; Rodgers et al., 2014; SalazarMora, 2008). Así, escuchar comentarios sobre el peso, ver publicidad que promueve la delgadez y ver personas que representan modelos sociales sumamente delgados desemboca en una intranquilidad por la cantidad de peso a perder, ya que se impone el estar acorde con el canon de belleza socialmente aceptado (Rodgers et al., 2014).

Y por último, en el tercer modelo, que refiere al malestar corporal, se encontró una relación con los mensajes verbales, la publicidad y las situaciones sociales, lo cual explica el hecho de que los mensajes recibidos del entorno social influyen en el tipo de publicidad que se ve, ya que con estos se promueve que se preste más atención o que se participe en situaciones sociales que tengan que ver con una imagen corporal ideal. La combinación de estos factores da como resultado un malestar corporal, el cual, si se reafirma con el tiempo, puede llevar a la insatisfacción corporal (Saffon $\&$ Saldarriaga, 2014). Cabe mencionar que esto no ocurre cuando se relaciona el malestar corporal con los mensajes verbales, la publicidad y los modelos sociales, y tampoco cuando se intenta relacionar la insatisfacción corporal con los mensajes verbales, la publicidad y los modelos sociales; lo cual puede deberse a que las mujeres no creen que su imagen corporal pueda ser comparable con una modelo que aparece en algún tipo de publicidad, ya que están conscientes de las diversas modificaciones que se realizan para que una modelo luzca bien (Bailey \& Ricciardelli, 2010).

Por lo anterior, se concluye que las situaciones sociales tienen un gran impacto en la percepción de las mujeres con respecto a su propia imagen corporal; lo que quiere decir que se pudo evidenciar que las influencias sociales son motivo tanto de preocupación por el cuerpo como de malestar e insatisfacción corporal. Por ello, es necesario que se realicen intervenciones preventivas en las que se tenga como objetivo el aumento de la resiliencia, el cambio en los esquemas cognitivos y el desarrollo de conductas saludables que permitan disminuir el impacto de los estándares de belleza socialmente aceptados (Espósito, 2015).

Finalmente, como limitaciones del estudio se tienen el tamaño de la muestra y que la participación de mujeres adolescentes fue escasa. Se sugiere que para futuras investigaciones se consideren medidas antropométricas.

\section{Conflicto de intereses}

Los autores declaran no tener ningún tipo de conflicto de intereses.

\section{Referencias}

Acosta, M., \& Gómez, G. (2003). Insatisfacción corporal y seguimiento de dieta. Una comparación transcultural entre adolescentes de España y México. International Journal of Clinical and Health Psychology, 3(1), 9-21. Recuperado de http://www.redalyc.org/articulo.oa?id=33730101

Amaya, A., Álvarez, G., \& Mancilla, J. (2010). Insatisfacción corporal en interacción con autoestima, influencia de pares y dieta restrictiva: Una revisión. Revista Mexicana de Trastornos Alimentarios, 1(1), 76-89. Recuperado de http://www.scielo.org.mx/scielo.php?script=sci_arttext\&pi $\mathrm{d}=\mathrm{S} 2007-15232010000100008$

Bailey, S., \& Ricciardelli, L. (2010). Social comparisons, appearance related comments, contingent self-esteem and their relationships with body dissatisfaction and eating disturbance among women. Eating Behaviors, 11(2), 107-112. doi: https://dx.doi.org/10.1016/j.eatbeh.2009.12.001

Balantekin, K., Birch, L., \& Savage, J. (2018). Family, friend, and media factors are associated with patterns of weightcontrol behavior among adolescent girls. Eating and Weight Disorders-Studies on Anorexia, Bulimia and Obesity, 23(2), 215-223. doi: https://doi.org/10.1007/s40519-016-0359-4

Bearman, S. K., Presnell, K., \& Martínez, E. (2006). The skinny on body dissatisfaction: A longitudinal study of adolescent girls and boys. Journal of Adolescence, 35(2), 217-229. doi: https://dx.doi.org/10.1007/s10964-005-9010-9

Botella, L., Ribas, E., \& Ruiz, J. (2009). Evaluación psicométrica de la imagen corporal: validación de la versión española del Multidimensional Body Self Relations Questionnaire (MBSRQ). Revista Argentina de Clínica Psicológica, 18(3), 253-264. Recuperado de http://www.redalyc.org/articu lo.oa? id=281921775006

Brudzynski, L. R., \& Ebben, W. (2010). Body image as a motivator and barrier to exercise participation. International Journal of Exercise Science, 3(1), 14-24. Recuperado de https://digitalcommons.wku.edu/cgi/viewcontent. cgi?article $=1172 \&$ context $=$ ijes

Byrne, B. M. (2016). Structural Equation Modeling with AMOS Basic Concepts, Applications, and Programming (3. ${ }^{\text {a }}$ ed.). Nueva York: Routledge. doi: http://doi. org/10.4324/9781315757421

Caccavale, L. J., Farhat, T., \& Iannotti, R. J. (2012). Social engagement in adolescence moderates the association between weight status and body image. Body Image, 9(2), 221-226. doi: http://doi.org/.1016/j.bodyim.2012.01.001

Cash, T. F. (1990). The psychology of physical appearance: Aesthetics, attributes, and images. En T. F. Cash \& T. Pruzinsky (eds.), Body Images: Development, Deviance, and Change (pp. 51-79). Nueva York: Guilford Press.

Castrillón, D., Luna, I., Avendaño, G., \& Perez-Acosta, A. M. (2007). Validación del Body Shape Questionnaire (Cuestionario de la Figura Corporal) BSQ para la población colombiana. Acta Colombiana de Psicología, 10(1), 
15-23.Recuperadodehttp://www.redalyc.org/pdf/798/79810 103.pdf

Cooper, P. J., Taylor, M. J., Cooper, Z., \& Fairbum, C. G. (1987). The development and validation of the Body Shape Questionnaire. International Journal of Eating Disorders, 6(4), 485-494. doi: https://doi.org/10.1002/1098108 X(198707)6:4<485::AID-EAT2260060405>3.0. $\mathrm{CO} ; 2-\mathrm{O}$

Cruwys, T., Leverington, C., \& Sheldon, A. (2016). An experimental investigation of the consequences and social functions of fat talk in friendship groups. International Journal of Eating Disorders, 49(1), 84-91. doi: https://doi. org/10.1002/eat.22446

Escobedo, M., Hernández, J., Estebané, V., \& Martínez, G. (2016). Modelos de ecuaciones estructurales: Características, fases, construcción, aplicación y resultados. Ciencia y Trabajo, 18(55), 16-22. doi: http://dx.doi. org/10.4067/S0718-24492016000100004

Espósito, D. H. (2015). Influencia del modelo estético corporal prevalente e insatisfacción con la imagen corporal en estudiantes de escuela secundaria básica. Revista Mexicana de Trastornos Alimentarios, 6(2), 91-96. doi: https://doi. org/10.1016/j.rmta.2015.08.001

Fardouly, J., Pinkus, R., \& Vartanian, L. (2017). The impact of appearance comparisons made through social media, traditional media, and in person in women's everyday lives. Body Image, 20, 31-39. doi: https://doi.org/10.1016/j. bodyim.2016.11.002

Ferguson, C. J., Winegard, B. M., \& Winegard, B. (2011). Who is the fairest one of all? How evolution guides peer and media influences on female body dissatisfaction. Review of General Psychology, 15(1), 11-28. doi: https://doi. org/10.1037/a0022607

Ferguson, C., Muñoz, M., Garza, A., \& Galindo, M. (2014). Concurrent and prospective analyses of peer, television and social media influences on body dissatisfaction, eating disorder symptoms and life satisfaction in adolescent girls. Journal of Youth and Adolescence, 43(1), 1-14. doi: https:// doi.org/10.1007/s10964-012-9898-9

Francisco, R., Narciso, I., \& Alarcão, M. (2013). Parental influences on elite aesthetic athletes' body image dissatisfaction and disordered eating. Journal of Child and Family Studies, 22(8), 1082-1091. doi: https://doi.org/10.1007/ s10826-012-9670-5

Frederick, D. A., Forbes, G. B., Grigorian, K., \& Jarcho, J. M. (2007). The UCLA body project I: Gender and ethnic differences in self-objectification and body satisfaction among 2,206 undergraduates. Sex Roles, 57(5-6), 317-327. doi: https://doi.org/10.1007/s11199-007-9251-z

Gerbasi, M., Richards, L., Thomas, J., Agnew-Blais, J., Thompson-Brenner, H., Gilman, S., \& Becker, A. (2014). Globalization and eating disorder risk: Peer influence, perceived social norms, and adolescent disordered eating in
Fiji. International Journal of Eating Disorders, 47(7), 727737. doi: https://doi.org/10.1002/eat.22349

Gonçalves, S., \& Bedin, L. (2016). Bienestar, salud e imagen corporal de adolescentes brasileros: la importancia de los contextos familiares, de amistad y escolar. Universitas Psychologica, 14(4), 1399-1410. doi: https:// doi.org/10.11144/Javeriana.up14-4.bsica

Grogan, S. (2008). Body Image: Understanding Body Dissatisfaction in Men, Women and Children (2. ${ }^{\mathrm{a}}$ ed.). Nueva York: Routledge.

Herraiz-Serrano, C., Rodríguez-Cano, T., Beato-Fernández, L., Latorre-Prostigo, J. M., Rojo-Moreno, L., \& Vaz-Leal, F. J. (2015). Parental rearing and eating psychopathology. Actas Españolas de Psiquiatría, 43(3), 91-98. Recuperado de https://www.actaspsiquiatria.es/repositorio/17/95/ENG/1795-ENG-91-8-429121.pdf

James, L. R., Mulaik, S. A., \& Brett, J. M. (1982). Causal Analysis: Assumptions, Models and Data. Beverly Hills: Sage.

Jauregui, I., \& Bolaños, P. (2011). Body Image and quality of life in a Spanish population. International Journal of General Medicine, 4(1), 63-72. doi: https://doi.org/10.1016/j. semerg.2011.07.019

Jones, W. R., \& Morgan, J. F. (2010). Eating disorders in men: A review of the literature. Journal of Public Mental Health,9(2), 23-31. doi: http://dx.doi.org/10.5042/ jpmh.2010.0326

Kline, R. B. (2015). Principles and Practice of Structural Equation Modeling (4. ${ }^{\text {a }}$ ed.). Nueva York: Guilford Press.

Losada, A. V., Leonardelli, E., \& Magliola, M. (2015). Influencia sociocultural y los trastornos de la conducta alimentaria en adolescentes. Revista Electrónica de Psicología Iztacala, 18(1), 380-416. Recuperado de http://www.revistas.unam. mx/index.php/repi/article/view/51178/45862

Mancilla, A., Vázquez, R., Mancilla, J., Amaya, A., \& Álvarez, G. (2012). Insatisfacción corporal en niños y preadolescentes: Una revisión sistemática. Revista Mexicana de Trastornos Alimentarios, 3(1), 62-79. Recuperado de http:// www.scielo.org.mx/pdf/rmta/v3n1/v3n1a8.pdf

Mas-Manchón, L., Rodríguez-Bravo, A., Montoya-Vilar, N., Morales-Morante, F., Lopes, E., ... Grau, A. (2015). Valores percibidos en la publicidad de alimentos por jóvenes con y sin trastornos de la conducta alimentaria. Salud Colectiva, 11(3), 423-444. doi: https://doi.org/10.18294/sc.2015.729

McDonald, R. P. (1999). Test Theory: A Unified Treatment. Mahwah: Lawrence Erlbaum Associates Publishers.

Moreno-Murcia, J., Marcos-Pardo, P., \& Huéscar, E. (2016). Motivos de práctica físico-deportiva en mujeres: diferencias entre practicantes y no practicantes. Revista de Psicología del Deporte, 25(1), 35-41. Recuperado de http://www.redalyc.org/articulo.oa?id=235143645005

Mulaik, S. A., James, L. R., Van Alstine, J., Bennett, N., Lind, S., \& Stilwell, C. D. (1989). Evaluation of 
goodness-of-fit indices for structural equation models. Psychological Bulletin, 105(3), 430-445. doi: https://doi. org/10.1037/0033-2909.105.3.430

Neziroglu, F., Khemlani-Patel, S., \& Veale, D. (2008). Social learning theory and cognitive behavioral models of body dysmorphic disorder. Body Image, 5(1), 28-38. doi: https:// doi.org/10.1016/j.bodyim.2008.01.002

Ramírez, M., Godoy, D., Vázquez, M., Lara, R., Navarrón, E., ... Jiménez, M. (2015). Imagen corporal y satisfacción corporal en adultos: Diferencias por sexo y edad. Revista Iberoamericana de Psicología del Ejercicio y el Deporte, 10(1), 63-68. Recuperado de http://www.redalyc.org/articulo.oa? id $=311132628008$

Raich, R. M., Mora, M., Soler, A., Ávila, C., Clos, I., \& Zapater, L. (1996). Adaptación de un instrumento de evaluación de la insatisfacción corporal. Clínica y Salud, 7(1), 51-66. Recuperado de https://journals.copmadrid.org/clysa/art/ f2217062e9a397a1dca429e7d70bc6ca

Rodríguez, S., \& Cruz, S. (2008). Insatisfacción corporal en adolescentes latinoamericanas y españolas. Psicothema, 20(1), 131-137. Recuperado de http://www.psicothema. com/pdf/3438.pdf

Rodríguez, M. N., \& Ruiz, M. A. (2008). Atenuación de la asimetría y de la curtosis de las puntuaciones observadas mediante transformaciones de variables: Incidencia sobre la estructura factorial. Psicológica, 29(2), 205-227. Recuperado de http://www.redalyc.org/pdf/169/16929206.pdf

Rodgers, R., Paxton, S., \& McLean, S. A. (2014). A biopsychosocial model of body image concerns and disordered eating in early adolescent girls. Journal of Youth and Adolescence, 43(5), 814-823. doi: https://doi.org/10.1007/ s10964-013-0013-7

Saffon, S., \& Saldarriaga, L. M. (2014). La internalización del ideal de delgadez: Factor de riesgo de alteraciones de la imagen corporal y los hábitos de alimentación, generado por la influencia de pares. Revista de Psicología Universidad de Antioquia, 6(1), 75-90.Recuperado de http://pepsic.bvsa lud.org/pdf/rpsua/v6n1/v6n1a06.pdf

Salazar-Mora, Z. (2008). Adolescencia e imagen corporal en la época de la delgadez. Revista Reflexiones, 87(2), 67-80. Recuperado de http://www.redalyc.org/ pdf/729/72912555004.pdf
Tabachnick, B., \& Fidell, L. S. (2013). Using Multivariate Statistics (6. ${ }^{\mathrm{a}}$ ed.). Londres: Pearson Education.

Thompson, B., Diamond, K. E., McWilliam, R., Snyder, P., \& Snyder, S. W. (2005). Evaluating the quality of evidence from correlational research for evidence-based practice. Exceptional Children, 71(2), 181-194. doi: https://doi. org/10.1177/001440290507100204

Toro, J., Salamero, M., \& Martínez, E. (1994). Assessment of sociocultural influences on the aesthetic body shape model in anorexia nervosa. Acta Psychiatrica Scandinavica, 89(3), 147-151. doi: https://doi.org/10.1111/j.1600-0447.1994. tb08084.x

Trejo-Ortíz, P., Mollinedo, F., Araujo, R., Valdez-Esparza, G., \& Sánchez, M. (2016). Hábitos de actividad física y cánones de imagen corporal en estudiantes universitarios. Revista Cubana de Medicina General Integral, 32(1), 72-82. Recuperado de http://scielo.sld.cu/pdf/mgi/v32n1/ mgi09116.pdf

Vaquero-Cristóbal, R., Alacid, F., Muyor, J. M., \& LópezMiñarro, P. A. (2013). Imagen corporal: Revisión bibliográfica. Nutrición Hospitalaria, 28(1), 27-35. doi: https:// dx.doi.org/10.3305/nh.2013.28.1.6016

Vázquez, R., Álvarez, G., \& Mancilla, J. (2000). Consistencia interna y estructura factorial del Cuestionario de Influencia de los Modelos Estéticos Corporales (CIMEC), en población mexicana. Salud Mental, 23(6), 18-24. Recuperado de http://www.redalyc.org/pdf/582/58262303.pdf

Vázquez, R., Galán, J., López, X., Alvarez, G., Mancilla, J., Caballero, A., \& Unikel, C. (2011). Validez del Body Shape Questionnaire (BSQ) en mujeres mexicanas. Revista Mexicana de Trastornos Alimentarios, 2(1), 42-52. Recuperado de http://www.scielo.org.mx/pdf/rmta/v2n1/ v2n1a5.pdf

Wasylkiw, L., \& Williamson, M. E. (2013). Actual reports and perceptions of body image concerns of young women and their friends. Sex Roles, 68(3-4), 239-251. doi: https://doi. org/10.1007/s11199-012-0227-2 